\title{
Recognition of Pole Piece Defects of Lithium Battery Based on Sparse De- composition
}

\author{
Gong Chen ${ }^{*}$, Xilu Lou, Chunxiang Li, Xifang Zhu ${ }^{*}$ Xu Cheng and Qingquan Xu
}

Changzhou Institute of Technology, Changzhou Key Laboratory of Optoelectronic Materials and Devices, Changzhou, Jiangsu, 213022, China

\begin{abstract}
To study surface denoising of lithium battery film to extract feature effectively. The best atomic function by sparse decomposition is acquired by iteration under added noise, gaussian noise, salt and pepper noise, additive and multiplicative noise. Terminating iteration value is got by observation and used to filter under specific background noise. Experiment shows sparse decomposition denoising performance is better than the median filter, sparse decomposition is good for detection of lithium battery film defects.
\end{abstract}

Keywords: Sparse decomposition, lithium battery film, median filter, defect.

\section{INTRODUCTION}

The application of lithium battery is not as good as it gets old. Battery used for automobile are composed of battery pack from a large number of single battery, and its service life is more than 2000 times. When composed of more than one battery and consistent highly in performance, the battery pack shows long service cycle close to the single. Considering equipment and technology of the lithium manufacturer's production are not mature in China, the quality of the product is affected. Surface defect is the important factor for the quality lithium battery film. Therefore, automatic detection of surface quality of thin film based on machine vision technology has gradually become the importance of research.

During production of lithium battery film, the defects are often represented by various sources in the process. So the problem can be confirmed by accurate and effective extraction of features to improve production technology. The lithium battery film image based on image enhancement technology aims to highlight the main feature of defects, but general algorithms usually focuses on median filtering [1], histogram equalization [2], area average [3], spatial filtering [3-6] and frequency domain and wavelet filtering technology [3].

Sparse decomposition is a new kind of signal and image decomposition method [7], It has extremely broad prospect in signal and image compression, denoising, signal analysis and signal recognition [8]. Under the condition lack of statistical noise feature, sparse decomposition algorithm can not only select appropriate base function adaptively to accomplish the decomposition of signal, but also capture the original feature of the original signal from redundant dictionary feature.
Lithium battery film images enhancement technology is present in this paper, including the application of sparse decomposition and comparison with the median filter technology. Lithium battery film defects from added noise, gauss noise, salt and pepper noise are de-noised by sparse decomposition method, then de-noised by median filter under the same background, finally, compared with the median filtering results.

\section{SPARSE DECOMPOSITION OF IMAGE SIGNAL BASED ON MATCHING PURSUIT ALGORITHM}

Matching pursuit algorithm(MP) is an iterative algorithm selecting the most matching atom for tracing the timefrequency structure of signal from highly redundant overcomplete dictionary. From the de-noising, defect target has small fluctuation of structure and flat distribution, besides it has certain structure whose property is as same as atom. However, there is bigger for the local fluctuation of noise which is the randomly relevant and lack of structure characteristic. If meaningful atom can be extracted from noisy image, extracted part is defect target or is noise target. During MP algorithm, signals or largest inner product atoms of residual will be chosen. Firstly flat defect signals comparatively are extracted, then local noise are extracted gradually. Sparse decomposition is the process of tracking and extracting atomic vector best matching the original signal and its residual signal. The atomic vector is the defect distribution. According to different noise background iteration terminal value conditions, we use artificial observation as empirical value.

The step is including in training and de-noising :

(1) Defining a over-complete dictionary from Hilbert Space $D=\left\{g_{r_{m}}(x, y)\right\}(m=0,1, \cdots, M-1) M$ is the iteration terminal value, $\left\|g_{r_{m}}(t)\right\|=1$. Selected atom in the dictionary is as the following : 


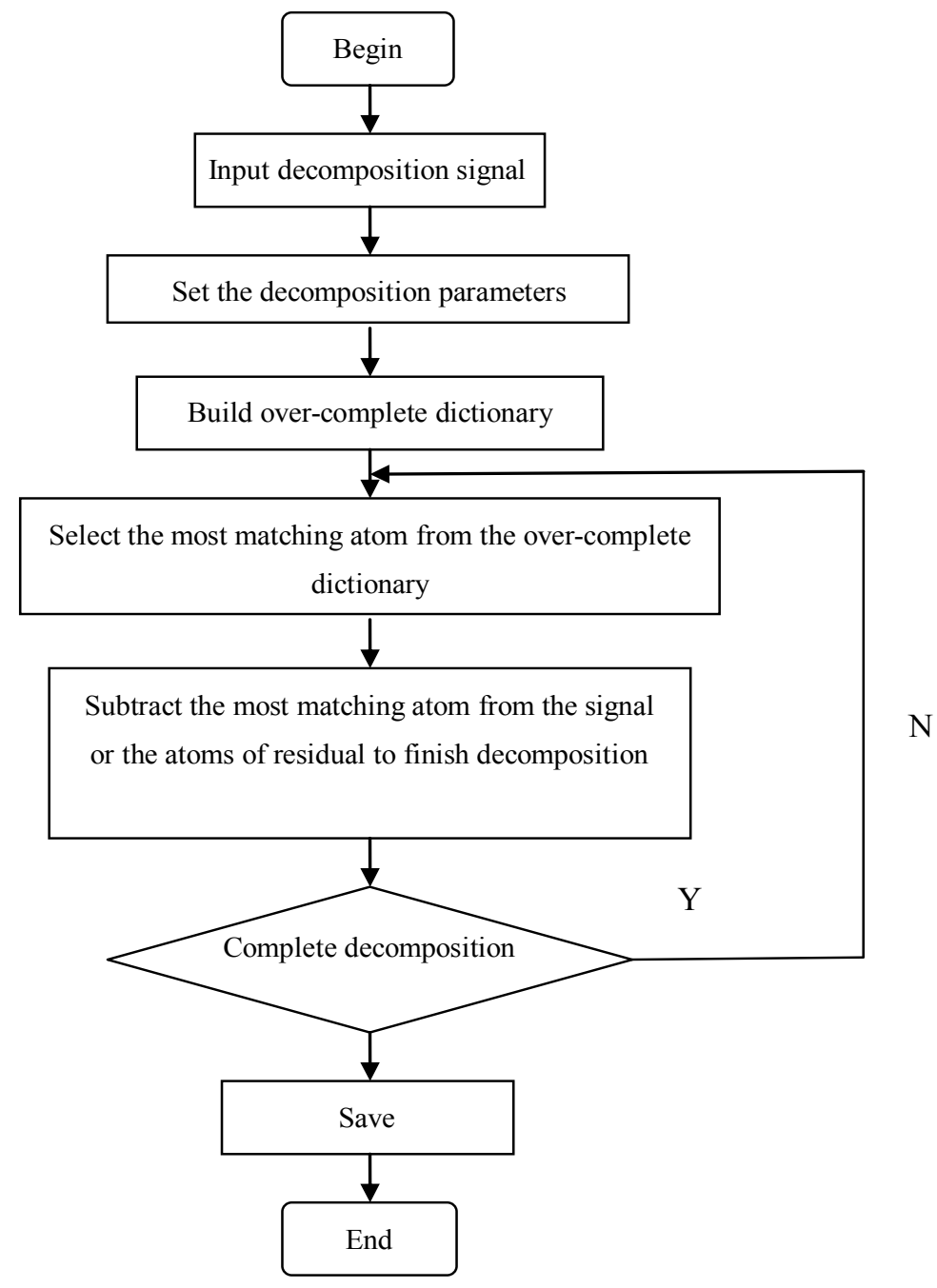

Fig. (1). Flow Chart of Sparse Decomposition Based on MP.

$g_{\gamma_{m}}(x, y)=\exp \left(-\frac{\left(x_{0}^{2}+v^{2} y_{0}^{2}\right)}{2 \sigma^{2}}\right) \times \cos \left(\frac{2 \pi}{\lambda} x_{0}\right)$

From Eqs. $1, x_{0}=x \cos \theta+y \sin \theta, y_{0}=-x \sin \theta+y \cos \theta$, $x=1,2, \mathrm{~L} T_{1}, y=1,2, \mathrm{~L} T_{2}, \mathrm{~T} 1$ is the atomic signal width, T2 is the atomic signal height, $\gamma=(\sigma, v, \lambda, \theta)$ is the timefrequency parameter which represents respectively the atomic expansion, distance, frequency and phase.

(2) Suppose specific noisy image signal is $z_{N}(x, y)$ and $z_{N}(x, y)=R_{0} z_{N}, N$ is the number of specific noise including Random noise, Gaussian noise, Salt and Pepper noise and Addition-multiplication noise, $R_{0} z_{N}$ is the original residual.

(3) Selecting the most matching atom $g_{\gamma_{o}}(x, y) \in D$ by MP which maximizes the $\left|\left\langle R_{0} z_{N}, g_{\gamma_{0}}(x, y)\right\rangle\right|$ and obtaining residual is $R_{1} z_{N}=R_{0} z_{N}-\left\langle R_{0} z_{N}, g_{\gamma_{0}}(x, y)\right\rangle g_{\gamma_{0}}(x, y)$. Selecting the most matching atom $g_{\gamma_{o}}(x, y) \in D$ by MP once again which maximizes the $\left|\left\langle R_{1} z_{N}, g_{\gamma_{1}}(x, y)\right\rangle\right|$ and obtaining residual is $R_{2} z_{N}=R_{1} z_{N}-\left\langle R_{1} z_{N}, g_{\gamma_{1}}(x, y)\right\rangle g_{\gamma_{1}}(x, y), \quad \mathbf{L} \mathbf{L}$, $R_{m} z_{N}=R_{m-1} z_{N}-\left\langle R_{m-1} z_{N}, g_{\gamma_{m-1}}(x, y)\right\rangle g_{\gamma_{m-1}}(x, y)$.

(4) From the above steps, iterative termination value $M$ is obtained by observation under different noise.

(5) Finally defect image signal $z z(x, y)=$ $\sum_{m=0}^{M}\left\langle R_{m} z_{N}, g_{\gamma_{m}}(x, y)\right\rangle g_{\gamma_{m}}(x, y)+R_{M+1} z_{N}$ is obtained by filter.

(6) Under the certain noise background, repeating Step (1) to (5) from Step (4) iterative termination empirical value to get defect image after the de-noising.

Fig. (1) shows the flowchart of the algorithm. Table 1 shows the various background noise characteristics. 
The original

Added noise image

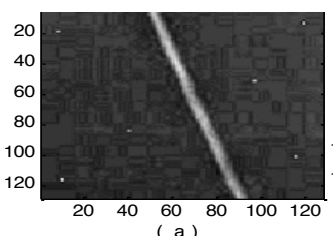

(a)
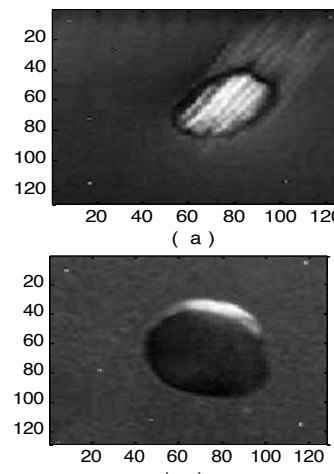

( a

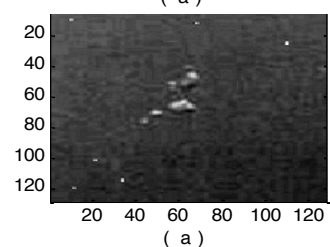

( a)
Began to

restore image
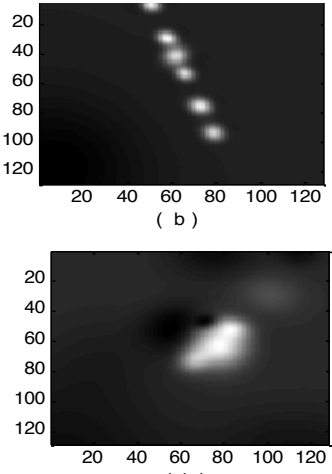

( b )

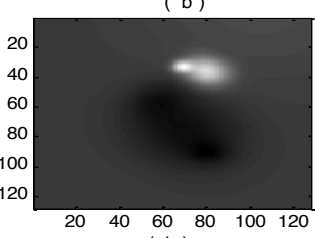

$(\mathrm{b})$

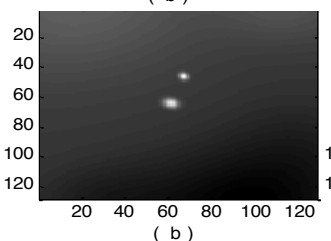

(a) Added Noise

The original

Added noise image
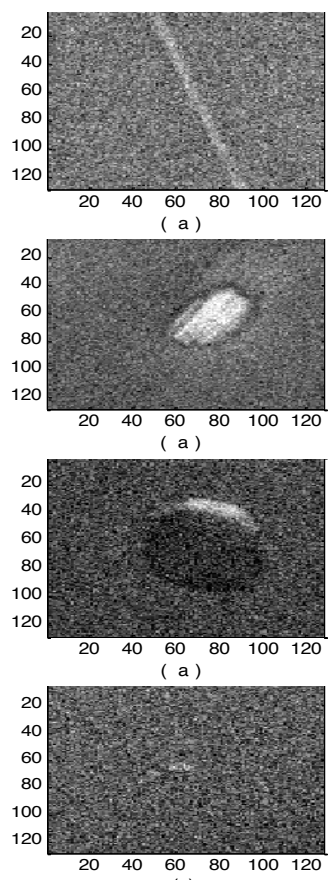

(a)
Began to

restore image

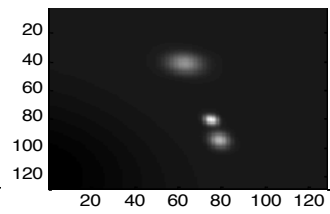

(b)

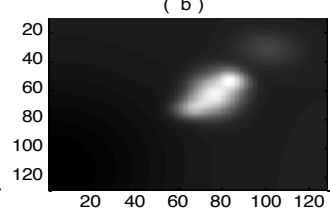

(b)
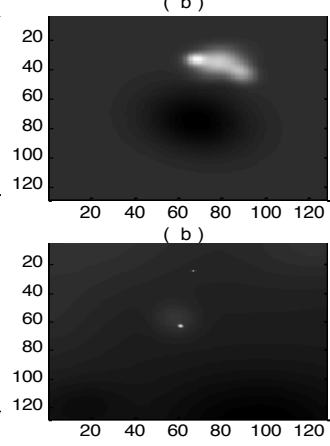

(b)
Defect restoration best image
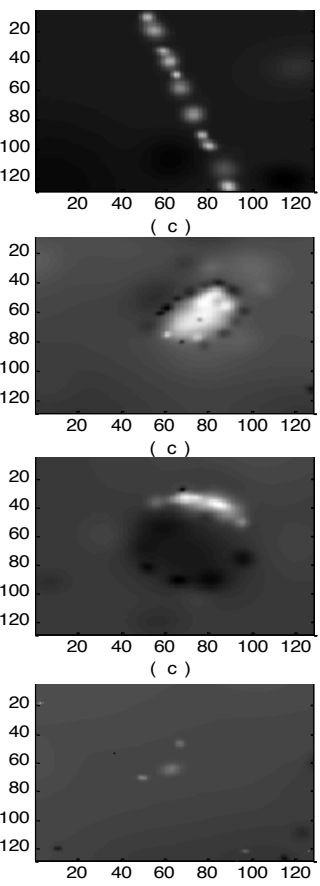

(c)
Appearing

spot noise
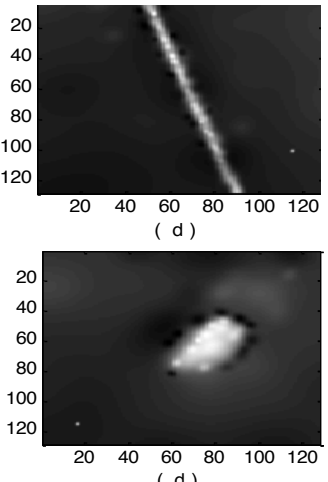

(d)
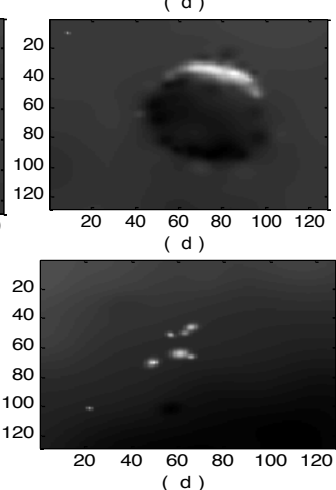

(b) Gaussian Noise 

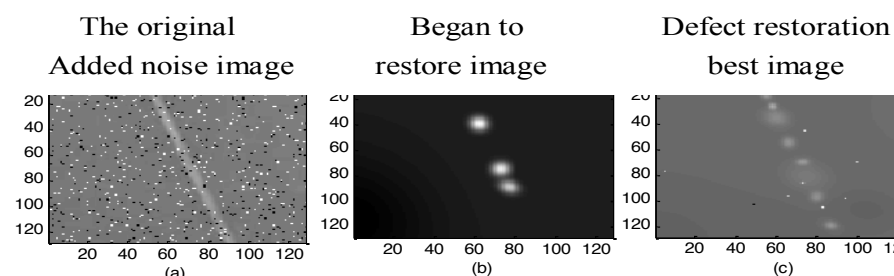
best image
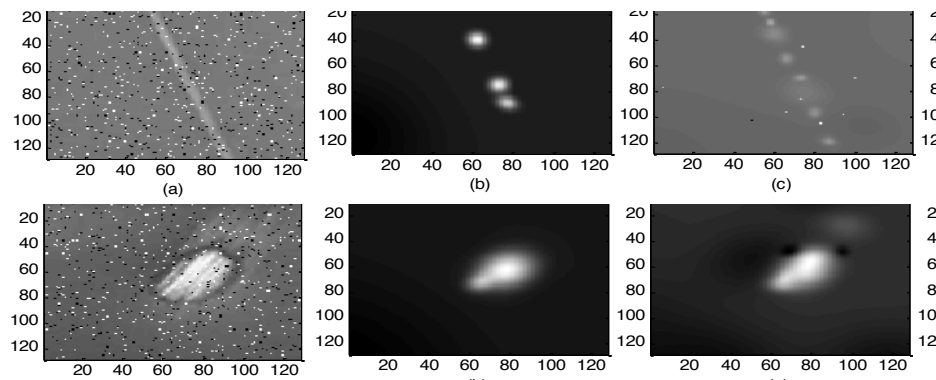

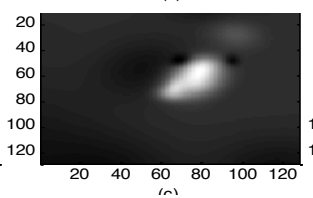

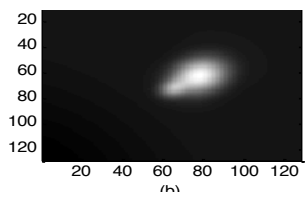

The original Added noise image
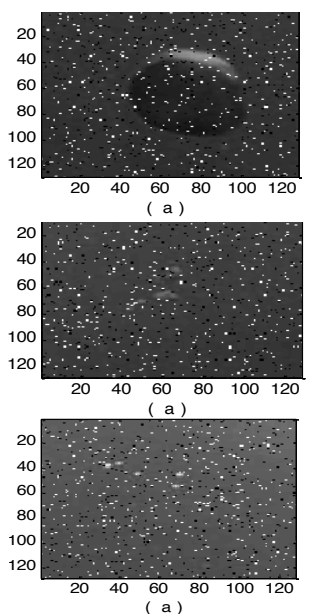

Appearing spot noise
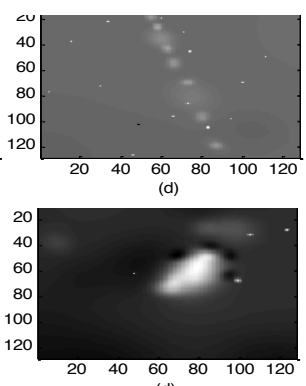

Defect restoration restore image best image
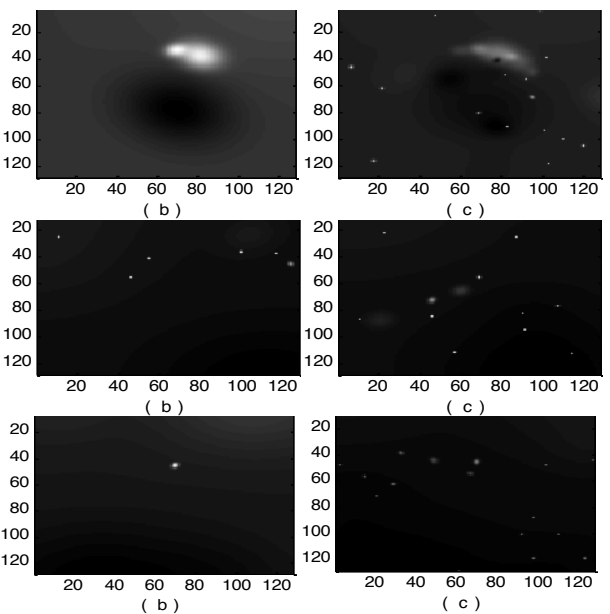

(c) Salt and Pepper Noise
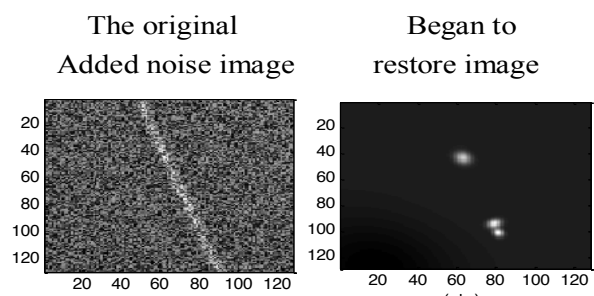
Defect restoration best image

Appearing spot noise
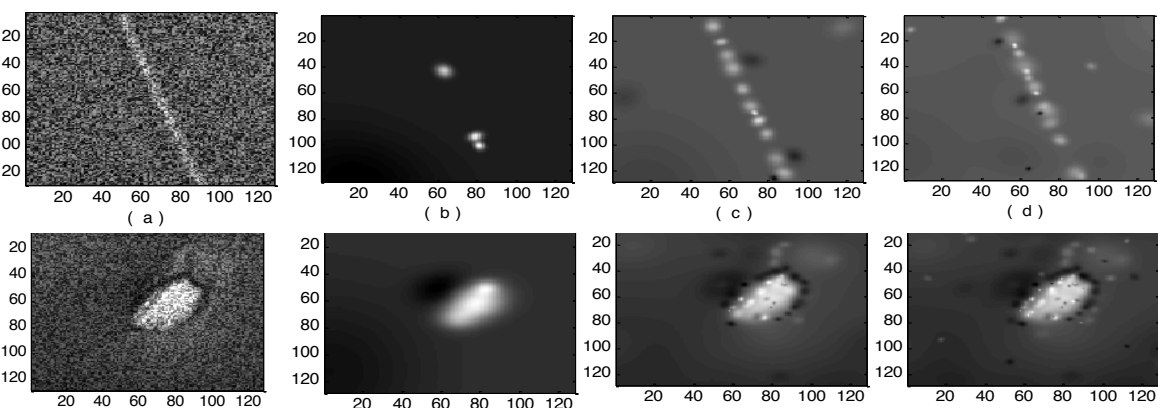

( a
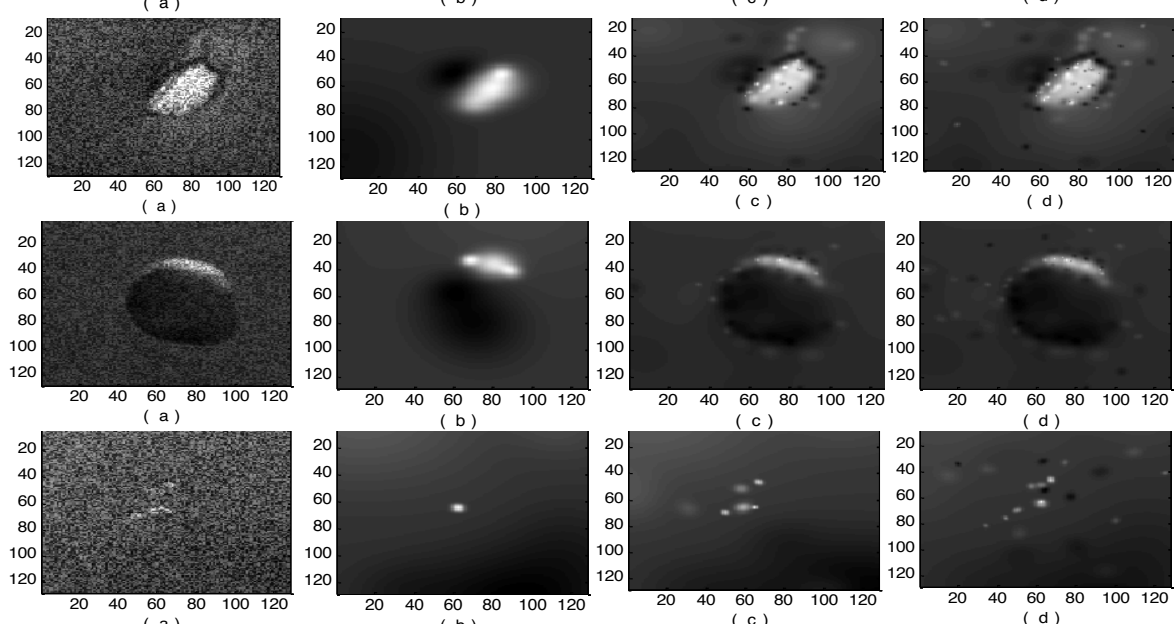

( a )
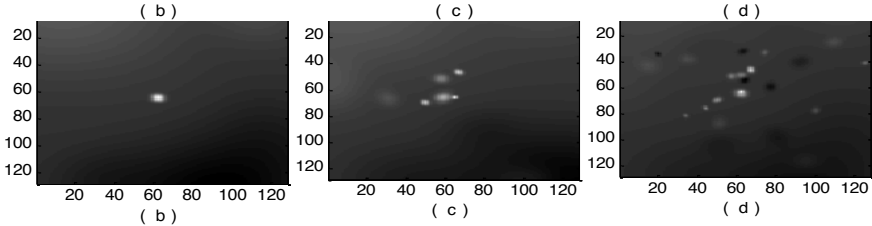

(d) Addition-multiplication Noise

Fig. (2). Defects under Different Noise Background Based on Sparse Decomposition. 
Table 1. Characteristics of Noise.

\begin{tabular}{|c|c|}
\hline Noise & Characteristics \\
\hline Added Noise & $\begin{array}{l}\text { It is added randomly in the lithium battery film defects on the graph. The purpose is to simulate influence from ran- } \\
\text { dom noise, such as particle、dust and insect on the camera. }\end{array}$ \\
\hline Gaussian Noise & $\begin{array}{l}\text { It is a random noise whose the probability density function comply with Gaussian distribution. The purpose is to simu- } \\
\text { late influence from light source. }\end{array}$ \\
\hline Salt and Pepper Noise & $\begin{array}{l}\text { Salt and Pepper noise is black and white spot noise. The purpose is to simulate influence from transmission and pro- } \\
\text { cess of lithium battery film defects image by CDD. }\end{array}$ \\
\hline Addition Multiplication Noise & $\begin{array}{l}\text { Addition Multiplication noise is produced by random scattering point. The purpose is to simulate influence from dy- } \\
\text { namic lithium battery film defects image by CDD. }\end{array}$ \\
\hline
\end{tabular}

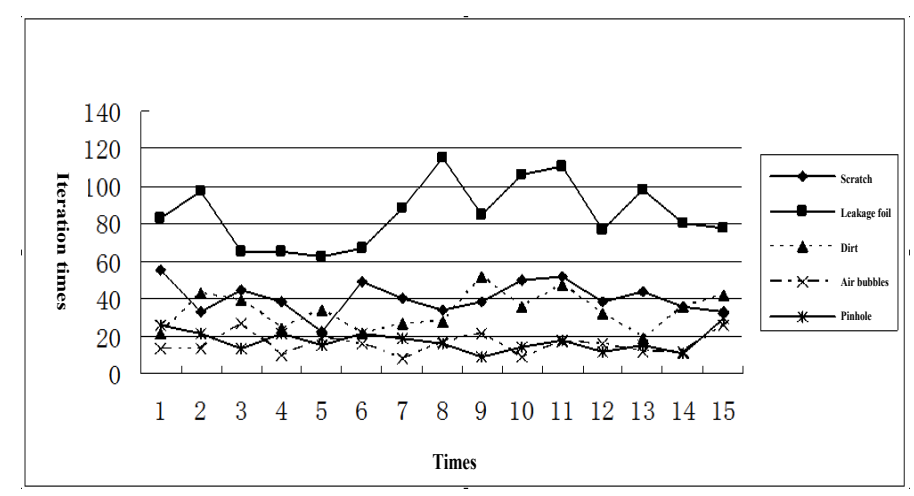

Fig. (3). Best Iteration Times under Added Noise.

\section{SIMULATION RESULTS}

The image of lithium battery film defects is set to $128 \mathrm{x}$ 128 pixels.

\subsection{Denoising of Film Defects with Add Noise}

Defined as $(i, j)$ by the ith row and the jth column.

Fig. (2a)(1.1) is the original man-made added noise image. Fig. (2a)(1.2) shows the reduction image by sparse decomposition. Fig. (2a)(1,3) is the best restore image of scratch defect and Fig. (2a) $(1,4)$ is the original man-made added noise image firstly. From Fig. (2a), during de-noising by sparse decomposition, image is restored from the region of scratch defect and from added noise in turn. 2-5th rows of Fig. (2a) are the results of leakage, dirt, bubble and needle defect. Experiment shows that it is effective obviously by sparse decomposition which is strong to defect reduction.

From Fig. (2b), it is obvious for scratch, dirt and leakage foil defect de-noising performance by sparse decomposition, but it is insufficient to needle and limit to bubble. Because of the smaller defects of pinhole and bubble, it can be found that the sparse decomposition denoising effect for small area defect is not good. The pinhole defect of denoising effect is better than that of bubble. Experiment shows that sparse decomposition algorithm for lithium battery film defects can restore origin figure from the defects purposefully.

From Fig. (2c), it's the best for salt and pepper noise of defect to be denoised by sparse decomposition, but it is barely for scratch to find defects and hardly for dirt, bubble and pinhole. Because a lot of white dots contained in salt and pepper noise influence denoising and white areas in the scratch defect and leakage foil defect are large and concentrated, sparse decomposition could be effective. At the same time pinhole defect with same white dots cannot be denoised, it also shows that sparse decomposition has better denoising effect for defects of larger area.

From Fig. (2d), It's not very good for each de-noised defect of lithium battery film. It is obvious for scratch, pinhole and leakage foil defect de-noising performance by sparse decomposition, but it is insufficient to dirt and limit to bubble. Experiment shows that sparse decomposition algorithm for lithium battery film defects can restore origin figure from the defects purposefully. Because of better denoising effect for Addition-Multiplication noise of lithium battery film defects by sparse decomposition and grey black image of addition-multiplication, compared with the image of salt and pepper noise that contains a large number of white dots, it proves that sparse decomposition has better denoising performance for the white part.

\subsection{Effect of Iteration Times and the Noise Area on the Performance of Sparse Decomposition}

In order to study the relationship between the specific noise and the number of iterations N, Fig. (3) shows the best number of iterations under added noise. It can be seen that iteration times depend on kind of noise and belongs to 

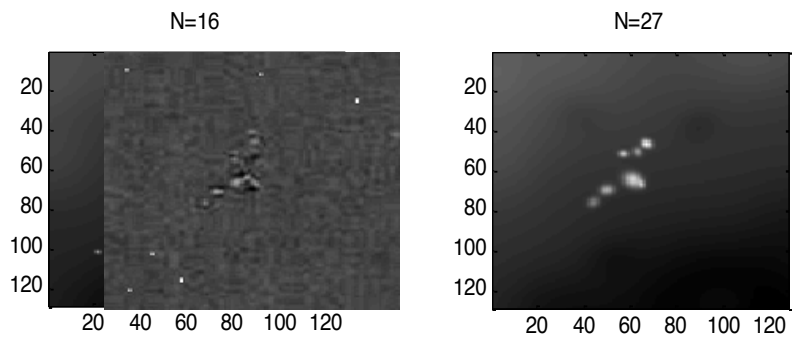

Fig. (4). The Best Restoration in Different Iteration Times.
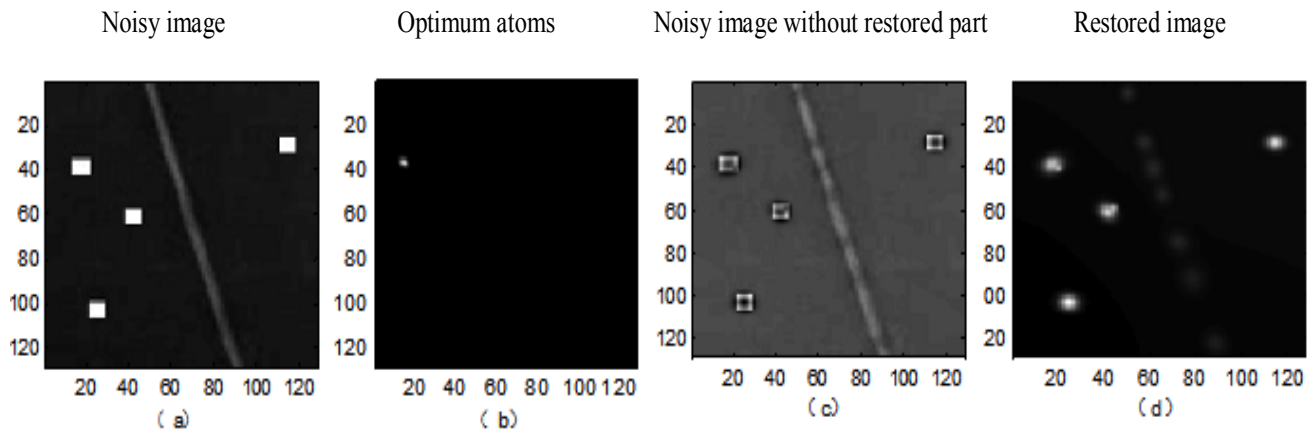

Fig. (5). Simulation Results of Larger Area Added Noise.

different range. Because the range of scratch, leakage foil, dirt is over the bubble and pinhole and $\mathrm{N}$ value of leakage foil reaches maximum, The greater is the defect area the bigger is the $\mathrm{N}$ value. Number of experiential iterations under different noise background can be set from variable $\mathrm{N}$.

When $\mathrm{N}$ is larger, defect image restored is more clear and vice versa. From Fig. (4), left figure is the image of bubble defects when $\mathrm{N}=27$ while right figure is $\mathrm{N}=16$. The clarity of defects in left is obviously better than the right, but the increasing of $\mathrm{N}$ value will lead to denoising incompletely. (As the noise left foot).

Considered sparse decomposition algorithm influenced by the area of spot noise, if the area is too large, the spot noise is restored firstly to affect deoising. From Fig. (5), the spot noise and the defect are restored alternately.

\subsection{Comparison between the Performance of Sparse De- composition and Median Filter}

\subsubsection{Denoising of the Added Noise}

From Fig. (6), Sparse decomposition is not effective for the added noise from lithium battery film instead of median filter.

\subsubsection{Denoising of Gaussian Noise, Salt and Pepper Noise and Addition-Multiplication Noise}

From above experiment, median filter can only get rid of the salt and pepper noise from lithium battery film defects, but sparse decomposition is better than median filter and can remove the added noise, Gaussian noise and Additionmultiplication noise. Thus the range of sparse decomposition is wider than one of median filter. This algorithm shows the dynamic process of denoising and has a strong targeted for the restore of defects. Therefore sparse decomposition is better than median filter.

\section{CONCLUSION}

For image enhancement of lithium battery film defects, the paper mainly studies the application of sparse decomposition in lithium battery film defects denoising and comparison between sparse decomposition and median filter. Defects of lithium batteries film with Added noise, Gaussian noise, Salt and Pepper noise and Addition-multiplication noise are removed by sparse decomposition and median filter. At last sparse decomposition and median filter are compared. Experiments show that, the performance of sparse decomposition is better than median filter, sparse decomposition has good ability to restore lithium battery film defects and remove the noise to identify the lithium battery film defects.

\section{CONFLICT OF INTEREST}

The authors confirm that this article content has no conflicts of interest.

\section{ACKNOWLEDGEMENTS}

This work was financially supported by the National Natural Science Foundation Cooperative Innovation 

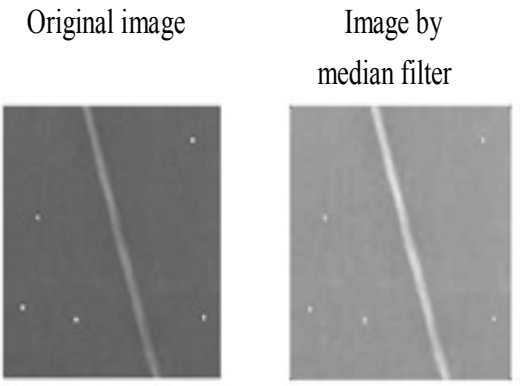

(a)
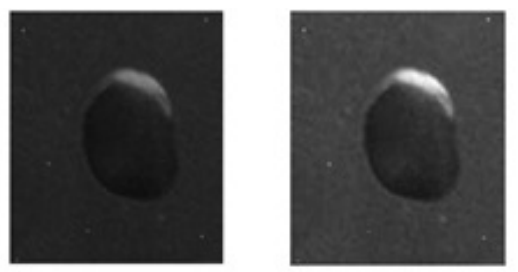

(c)

Original image

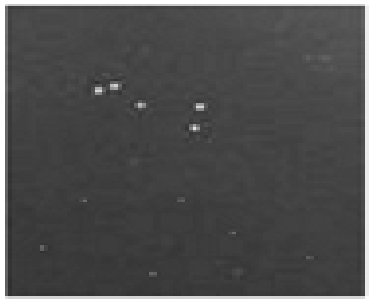

Original image sparse decomposition

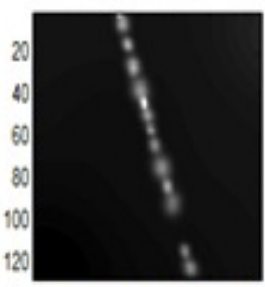

$20 \quad 4060 \quad 80 \quad 100120$

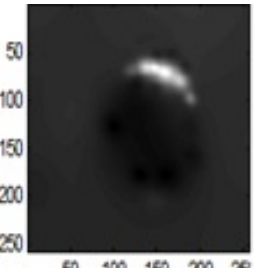

$50 \quad 100 \quad 150 \quad 200 \quad 26$
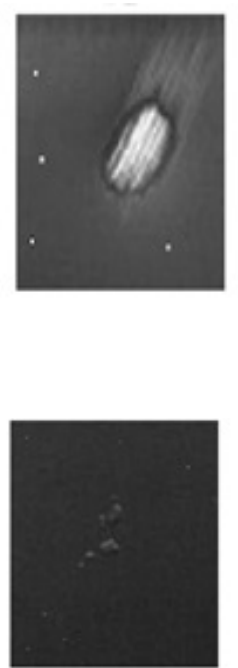

median filter

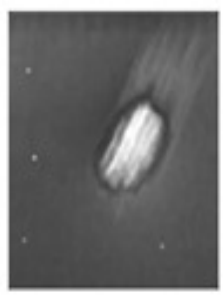

(b)

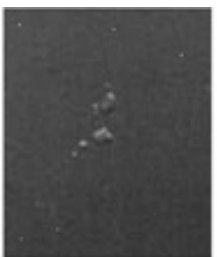

(d)
Image by sparse decomposition

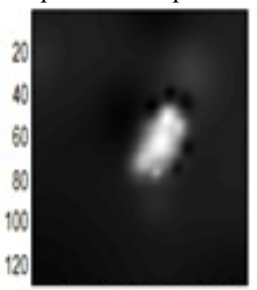

2010 4080100120

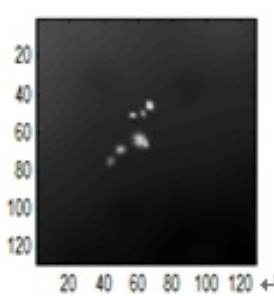

Image by sparse decomposition

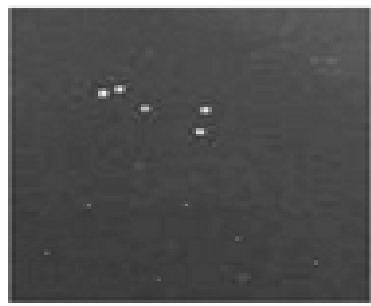

(e)

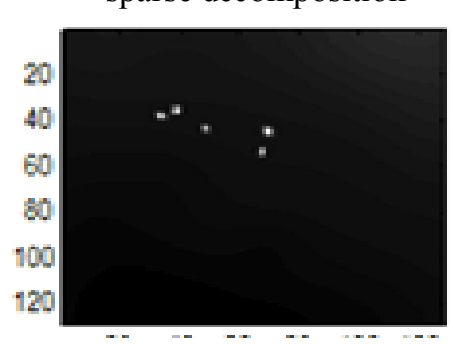

Fig. (6). Comparison under Different Defect Denoising.

(61475027); Cooperative Innovation Fund Project of Jiangsu Province of China (BY2014040); Natural Science Foundation of Jiangsu Province of China (BK20130245); Changzhou Key Laboratory of Optoelectronic Materials and Devices(20130694).

\section{REFERENCES}

[1] X. Liu, "Design and Research on Lithium-ion Battery Pole Piece Inspection System Based on Machine Vision," South China University of Technology, 2010.

[2] H. Zhao, "Research on Surface Defect Inspection of Solar Cell Based on Machine Vision," Yanshan University, 2011.

[3] L. Wang, "A Study of Inspection of Cell Surface Defects Based on Machine Vision," University of Science and Technology of China, 2011.
[4] Y. Wang, B. Wei, and K. Ning, "Topology acquisition and contact analysis of image threshold of Cu-based sintered material," Surface Technology, vol. 43, pp. 40-43, 2001.

[5] X. Dai, and G. Wang, "Design and realization for extraction of defect image on the surface of spare parts based on pattern recognition," Surface Technology, vol. 40, pp.109-112, 2011.

[6] J. Gang, F. Han, and L. Tang, "The research for recognizing algorithm of image pattern on corrosion character of electrodeposited coating," Surface Technology, vol.30, pp.23-26, 2001.

[7] J. Wang, and Z. Yin, "Sparse signal and image decomposition and Preliminary Application," Southwest Jiao Tong University press, 2006.

[8] S. Mallt, and Z. Zhang, "Matching pursuits with time-frequency dictionaries," IEEE Transactions on Signal Processing, vol. 41, pp. 3397-3415, 1993.

Received: June 02, 2015

Revised: August 02, 2015

Accepted: September 05, 2015

(C) Yuan et al.; Licensee Bentham Open.

This is an open access article licensed under the terms of the Creative Commons Attribution Non-Commercial License (http://creativecommons.org/licenses/by$\mathrm{nc} / 3.0 /$ ) which permits unrestricted, non-commercial use, distribution and reproduction in any medium, provided the work is properly cited. 